\title{
An Exact Analytical Solution of the Strong Shock Wave Problem in Nonideal Magnetogasdynamics
}

\author{
S. D. Ram, ${ }^{1}$ R. Singh, ${ }^{2}$ and L. P. Singh ${ }^{2}$ \\ ${ }^{1}$ Department of Mathematics, Mata Sundri College, University of Delhi, Delhi 110002, India \\ ${ }^{2}$ Department of Applied Mathematics, Indian Institute of Technology (BHU), Varanasi 221005, India \\ Correspondence should be addressed to S. D. Ram; sdram.apm@gmail.com
}

Received 27 May 2013; Accepted 11 September 2013

Academic Editor: Miguel Onorato

Copyright (c) 2013 S. D. Ram et al. This is an open access article distributed under the Creative Commons Attribution License, which permits unrestricted use, distribution, and reproduction in any medium, provided the original work is properly cited.

\begin{abstract}
We construct the solutions to the strong shock wave problem with generalized geometries in nonideal magnetogasdynamics. Here, it is assumed that the density ahead of the shock front varies according to a power of distance from the source of the disturbance. Also, an analytical expression for the total energy carried by the wave motion in nonideal medium under the influence of magnetic field is derived.
\end{abstract}

\section{Introduction}

The propagation of shock waves, generated by a strong explosion in earth's atmosphere is of great interest both from mathematical and physical point of view due to its numerous applications in various fields. They result from a sudden release of a relatively large amount of energy; typical examples are lightening and chemical or nuclear explosions. Assume that we have an explosion, following which there may exist for a while a very small region filled with hot matter at high pressure, which starts to expand outwards with its front headed by a strong shock. The process generally takes place in a very short time after which a forward-moving shock wave develops, which continuously assimilates the ambient air into the blast wave. The study of strong shock wave problems has been of long interest for researchers in fields ranging from condensed matter to fluid dynamics due to its theoretical and practical importance. Practically, it is recognized that strong shock waves are excellent means for generating very highpressure, high temperature plasma at the center of explosion. Many authors, for example, Arora and Sharma [1], Sakurai $[2,3]$ and Rogers [4] have presented exact solutions for the problem of strong shock wave with spherical geometry, since the study of spherically symmetric motion is important for the theory of explosion in various gasdynamic regimes. Recently, Singh et al. $[5,6]$ presented an approximate analytical solution to the system of first order quasilinear partial differential equations that govern a one dimensional unsteady planar and nonplanar motion in ideal and nonideal gases, involving discontinuities.

The study of interaction between gasdynamic motion of an electrically conducting medium and a magnetic field has been of great interest to scientists and engineers due to its application in astrophysics, geophysics, and interstellar gas masses. Taylor [7] obtained exact solution of the equations governing the motion in a gas generated by a point explosion. Singh et al. [8] studied the influence of the magnetic field upon the collapse of the cylindrical shock wave problem. Menon and Sharma [9] investigated the influence of magnetic field on the process of steepening and flattening of the characteristic wave front in a plane and cylindrically symmetric motion of ideal plasma. Oliveri and Speciale [10] used the substitution principle to obtain an exact solution for unsteady equation of perfect gas. Murata [11] obtained the exact solution for the one dimensional blast wave problem with generalized geometry. Singh et al. [12] have used quasisimilar theory to construct an analytical solution for the strong shock wave problem with generalized geometries in a nonideal gas satisfying the equation of state of the Van der Waals type.

The present paper aims to construct the closed form solution of the basic equations governing the one dimensional unsteady flows of a nonideal gas involving strong shock waves under the influence of transverse magnetic field. The basic configuration investigated here is that which arises when 
a transverse magnetic field is generated by a current of finite, constant strength passing along a straight wire of infinite length and either a shock or detonation wave propagates with uniform speed outwards from the wire into the ambient undisturbed gas at rest. Also, an expression for the total energy carried by the wave motion is obtained.

\section{Problem Formulation}

The basic equations for unsteady flow of a one dimensional gasdynamic motion may be written as $[8,12-14]$

$$
\begin{gathered}
\frac{\partial \rho}{\partial t}+u \frac{\partial \rho}{\partial r}+\rho\left(\frac{\partial u}{\partial r}+\frac{m}{r} u\right)=0 \\
\frac{\partial u}{\partial t}+u \frac{\partial u}{\partial r}+\frac{1}{\rho}\left(\frac{\partial p}{\partial r}+\frac{\partial h}{\partial r}\right)=0 \\
\frac{\partial p}{\partial t}+u \frac{\partial p}{\partial r}+a^{2} \rho\left(\frac{\partial u}{\partial r}+\frac{m}{r} u\right)=0 \\
\frac{\partial h}{\partial t}+u \frac{\partial h}{\partial r}+2 h\left(\frac{\partial u}{\partial r}+\frac{m}{r} u\right)=0
\end{gathered}
$$

where $\rho$ is the gas density, $u$ is the velocity along the $x$-axis, $p$ is the pressure, $h=\mu H^{2} / 2$ is the magnetic pressure with $H$ as the magnetic field strength, $\mu$ the magnetic permeability, $t$ is the time, $a=(\gamma p / \rho(1-b \rho))^{1 / 2}$ is the speed of sound, $r$ is the single spatial coordinate being either axial in flows with planer $(m=0)$ geometry or radial in cylindrically symmetric $(m=1)$ and spherically symmetric $(m=2)$ flows, and $\gamma$ is the constant specific heat ratio.

The system of (1)-(4) is supplemented with a Van der Waals equation of state of the form:

$$
p(1-b \rho)=\rho \Re T
$$

where $b$ is the Van der Waals excluded volume which is known in terms of the molecular interaction potential in high temperature gases, $T$ is the absolute temperature, and $\mathfrak{R}$ is the gas constant. It may be noted that the case $b=0$ corresponds to an ideal gas (ideal in the sense that the particle interactions are absent).

The propagation velocity of the shock front $U$ may be given as

$$
\frac{d R}{d t}=U
$$

where $R$ is the position of the shock front which is a function of time $t$.

\section{Rankine-Hugoniot Conditions}

The Rankine-Hugoniot relations, given by the principle of conservation of mass, momentum, and energy across the shock front may be expressed as $[5,6,15]$

$$
\begin{gathered}
\rho=\frac{\gamma+1}{(\gamma-1+2 \bar{b})} \rho_{a}, \\
u=\frac{2(1-\bar{b})}{\gamma+1} U, \\
p=\left\{\frac{2(1-\bar{b})}{\gamma+1}+\frac{2 C_{0}((\gamma-1) \bar{b}-\gamma)}{(\gamma-1+2 \bar{b})^{2}}\right\} \rho_{a} U^{2}, \\
h=\frac{C_{0}}{2}\left(\frac{(\gamma+1)}{(\gamma-1+2 \bar{b})}\right)^{2} \rho_{a} U^{2},
\end{gathered}
$$

where $\bar{b}=b \rho_{a}$ and $C_{0}=2 h_{a} / \rho_{a} U^{2}$ is the cowling number.

In the present problem the density $\rho_{a}$ is taken to vary according to the power law of radius of the shock front $R$, given as

$$
\rho_{a}=\rho_{0} R^{\lambda}
$$

where $\rho_{0}$ and $\lambda$ are constants. The constant $\lambda$ is determined in the subsequent analysis.

\section{Solution of the Strong Shock Wave Problem}

We construct a relation for the pressure in the flow field satisfying the Rankine-Hugoniot relations (7a)-(7d) as

$$
p=\left\{\frac{C_{0}(\gamma+1)(\bar{b}+\gamma)-(\gamma-1+2 \bar{b})^{2}}{(\bar{b}-1)(\gamma-1+2 \bar{b})}\right\} \rho u^{2} .
$$

Inserting (9) into (2) and (3) yields

$$
\begin{aligned}
& \frac{\partial u}{\partial t}+u \frac{\partial u}{\partial r}+\frac{\left\{C_{0}(\gamma+1)+4(1-\bar{b})\right\}(\gamma-1+2 \bar{b})}{8(1-\bar{b})^{2}} \\
& \quad \times\left(\frac{u^{2}}{\rho} \frac{\partial \rho}{\partial r}+2 u \frac{\partial u}{\partial r}\right)=0 \\
& \frac{\partial u}{\partial t}+u \frac{\partial u}{\partial r}+\frac{(\gamma-1)^{2}+(3 \gamma-1) \bar{b}}{2(\gamma-1)(1-\bar{b})} u\left(\frac{\partial u}{\partial r}+\frac{m}{r} u\right)=0 .
\end{aligned}
$$

Combining (10) and (4) and integrating the resulting equation we get

$$
S(t)=\rho u^{2-\alpha} r^{-m \alpha}
$$

where $S(t)$ is a arbitrary function of integration. Also, $\alpha$ is constants given as

$$
\alpha=\frac{4(1-\bar{b})\left\{(\gamma-1)^{2}+(3 \gamma-1) \bar{b}\right\}}{(\gamma-1)\left\{\left(C_{0}(\gamma+1)+4(1-\bar{b})\right)(\gamma-1+2 \bar{b})\right\}} .
$$


Using the solution (12), (1) takes the form

$$
\frac{(2-\alpha)}{u} \frac{\partial u}{\partial t}+(1-\alpha) \frac{\partial u}{\partial r}-(1+\alpha) \frac{m u}{r}-\frac{1}{S} \frac{d S}{d t}=0 .
$$

Solving (4) and (14), we have

$$
u=-\Omega \frac{r}{S} \frac{d S}{d t},
$$

where $\Omega=1 /\{\delta+m(\alpha+\delta)\}$ is a constant and $\delta$ given as

$$
\delta=1+\left(1-\frac{\alpha}{2}\right)\left(\frac{(\gamma-1)^{2}+\bar{b}(3 \gamma-1)}{(\gamma-1)(1-\bar{b})}\right) .
$$

Plugging the value of $u$ from (15) into (14), yields

$$
S(t)=S_{0} t^{-\eta}
$$

Here, $S_{0}$ is arbitrary constant and constant $\eta$ is given as

$$
\eta=\frac{(\alpha-2)}{(\alpha-1) \Omega+(\alpha+1) m \Omega-1} .
$$

Using the Rankine-Hugoniot relations (7a)-(7d) and (15) in (6) yield the analytical expression for the radius of the shock front as

$$
R(t)=t^{((\gamma+1) / 2)(\Omega \eta /(1-\bar{b}))} .
$$

With the help of Rankine-Hugoniot condition (7a)-(7d), we may determine the value of the constant $\lambda$ as

$$
\lambda=\frac{(\gamma+1)(m-1)-2(1-\bar{b})[(\alpha+1)(m+1)-2]}{(\gamma+1)} .
$$

Consequently, the analytical solution of the blast wave problem described in the prior section is given as

$$
\begin{aligned}
& u=\Omega \eta_{t}^{r}, \\
& \rho=\frac{1}{(\Omega \eta)^{(2-\alpha)}} S_{0} r^{(m+1) \alpha-2} t^{(2-\alpha-\eta)}, \\
& p=\left\{\frac{C_{0}(\gamma+1)(\bar{b}+\gamma)-(\gamma-1+2 \bar{b})^{2}}{(\bar{b}-1)(\gamma-1+2 \bar{b})}\right\} \\
& \times \frac{1}{(\Omega \eta)^{-\alpha}} S_{0} r^{(m+1) \alpha} t^{-(\alpha+\eta)}, \\
& h=\left\{\frac{\left(C_{0}(\gamma+1)+4(1-\bar{b})\right)(\gamma-1+2 \bar{b})}{4(1-\bar{b})^{2}}\right. \\
& \left.-\frac{C_{0}(\gamma+1)(\bar{b}+\gamma)-(\gamma-1+2 \bar{b})^{2}}{(\bar{b}-1)(\gamma-1+2 \bar{b})}\right\} \\
& \times \frac{1}{(\Omega \eta)^{-\alpha}} S_{0} r^{(m+1) \alpha} t^{-(\alpha+\eta)} .
\end{aligned}
$$

For a spherical motion of an ideal gas, the solutions (21) recover the results presented by some authors $[2,4,7,11]$.

\section{Behaviour of the Energy}

The total energy carried by the wave motion in nonideal medium under the influence of transverse magnetic field is expressed as [15]

$$
E=4 \pi \int_{0}^{R}\left\{\frac{1}{2} \rho u^{2}+\frac{(1-b \rho)}{\gamma-1} p+h\right\} r^{m} d r,
$$

where $E$ is the function of time and represents the sum of the kinetic and internal energy of the gas.

Using solutions (21) into (22), we have

$$
E(t)=\Pi t^{-(\alpha+\eta)+((m+1)(\gamma+1)(\alpha+1) \Omega \eta / 2(1-\bar{b}))} .
$$

The constant $\Pi$ appearing in the above relation may be expressed as

$$
\begin{aligned}
& \Pi=\frac{4 \pi}{(m+1)(\alpha+1)} \\
& \times\left\{1+\frac{C_{0}(\gamma+1)^{3}}{4(1-\bar{b})^{2}}\right. \\
& +\left(4 \bar{b}^{2}-\bar{b}\left(4+C_{0}+\left(C_{0}-4\right) \gamma\right)\right. \\
& \left.\left.+(\gamma-1)^{2}-C_{0}(\gamma+1)\right)\left((\gamma-1+2 \bar{b})^{2}\right)^{-1}\right\} \\
& \times \frac{S_{0}}{(\Omega \eta)^{-\alpha}} .
\end{aligned}
$$

\section{Result and Discussion}

The solution (21), which is an exact solution for nonideal magnetogasdynamic problems, obtained in the form of a power in the distance and time, is same as provided by [6-11] for strong and weak shock waves in ideal gases. However, for a planar and nonplanar nonideal magnetogasdynamic motion, the solutions (21) are a new exact solution in case of strong shock waves for arbitrary values of adiabatic index. From the solution equations (21), it is also clear that in the absence of magnetic field solutions coincides with the solution given by Singh et al. [5].

It may be noted here that the total energy carried by the wave remains constant in planar case of ideal gas, whereas in case of nonideal medium it varies with respect time $t$ and is given by (23). Also, due to increase in magnetic field strength total energy monotonically decreases with time $t$ and this process is slowed down due to the increase in Van der Waals excluded volume.

\section{Conclusion}

In the present paper, a new exact solution is derived for a strong shock wave problem in nonideal magnetogasdynamics 
with the density ahead of the shock which is varied as a power of the distance from the origin of the shock wave. The effect of coupling between the nonideal effects and magnetogasdynamics phenomena on the flow field is analyzed. The exact solution presented in (21) becomes the same results, for the absence of magnetic field, presented by Singh et al. [5]. The behavior of the total energy is also presented by (23).

\section{Acknowledgment}

R. Singh acknowledges the financial support from the UGC, New Delhi, India, under the SRF scheme.

\section{References}

[1] R. Arora and V. D. Sharma, "Convergence of strong shock in a Van der Waals gas," SIAM Journal on Applied Mathematics, vol. 66, no. 5, pp. 1825-1837, 2006.

[2] A. Sakurai, "On the propagation and structure of the Blast wave, I," Journal of the Physical Society of Japan, vol. 8, no. 5, pp. 662665, 1953.

[3] A. Sakurai, "On the propagation and structure of a Blast wave, II," Journal of the Physical Society of Japan, vol. 9, no. 2, pp. 256266, 1954.

[4] M. H. Rogers, "Analytic solutions for the Blast-waves problem with an atmosphere of varying density," Astrophysical Journal, vol. 125, pp. 478-493, 1957.

[5] L. P. Singh, S. D. Ram, and D. B. Singh, "Analytical solution of the Blast wave problem in a non-ideal gas," Chinese Physics Letters, vol. 28, no. 11, Article ID 114303, 2011.

[6] L. P. Singh, S. D. Ram, and D. B. Singh, "Exact solution of planar and nonplanar weak shock wave problem in gasdynamics," Chaos, Solitons \& Fractals, vol. 44, no. 11, pp. 964-967, 2011.

[7] J. L. Taylor, "An exact solution of the spherical Blast wave problem," Philosophical Magazine, vol. 46, pp. 317-320, 1955.

[8] L. P. Singh, S. D. Ram, and D. B. Singh, "The influence of magnetic field upon the collapse of a cylindrical shock," Meccanica, vol. 48, no. 4, pp. 841-850, 2013.

[9] V. V. Menon and V. D. Sharma, "Characteristic wave fronts in magnetohydrodynamics," Journal of Mathematical Analysis and Applications, vol. 81, no. 1, pp. 189-203, 1981.

[10] F. Oliveri and M. P. Speciale, "Exact solutions to the unsteady equations of perfect gases through Lie group analysis and substitution principles," International Journal of Non-Linear Mechanics, vol. 37, no. 2, pp. 257-274, 2002.

[11] S. Murata, "New exact solution of the Blast wave problem in gas dynamics," Chaos, Solitons and Fractals, vol. 28, no. 2, pp. 327330, 2006.

[12] L. P. Singh, S. D. Ram, and D. B. Singh, "Quasi-similar solution of the strong shock wave problem in non-ideal gas dynamics," Astrophysics and Space Science, vol. 337, no. 2, pp. 597-604, 2012.

[13] R. Courant and K. O. Friedrichs, Supersonic Flow and Shock Wave, Wiley, New York, NY, USA, 1948.

[14] G. B. Whitham, Linear and Non-Linear Waves, Wiley, New York, NY, USA, 1974.

[15] C. C. Wu and P. H. Roberts, "Structure and stability of a spherical shock wave in a van der Waals gas," Quarterly Journal of Mechanics and Applied Mathematics, vol. 49, no. 4, pp. 501-543, 1996. 

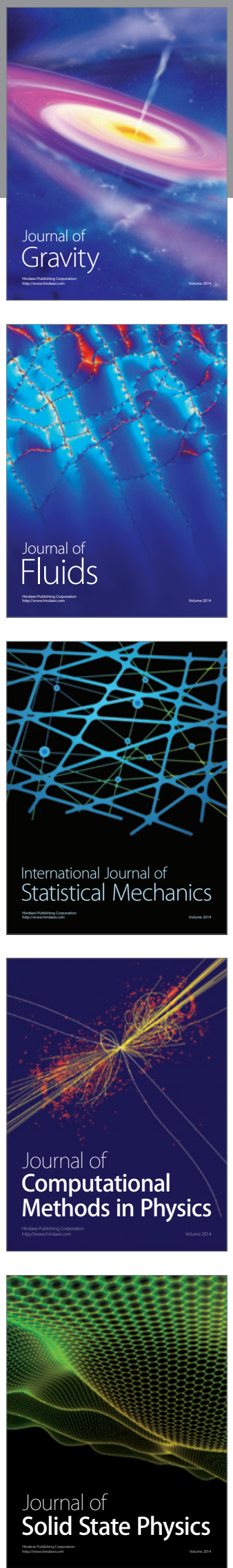

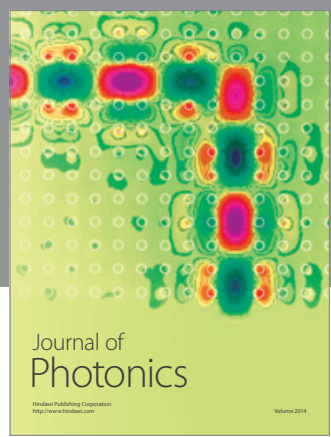

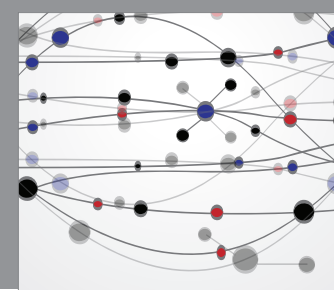

The Scientific World Journal

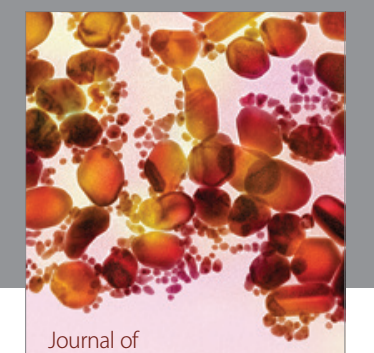

Soft Matter
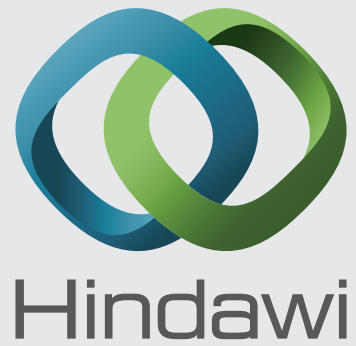

Submit your manuscripts at

http://www.hindawi.com
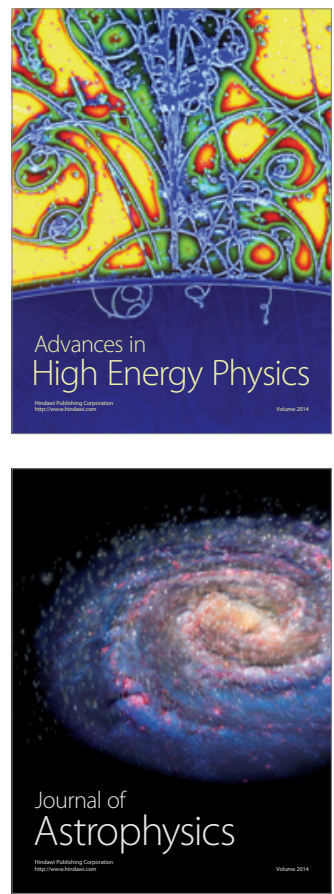
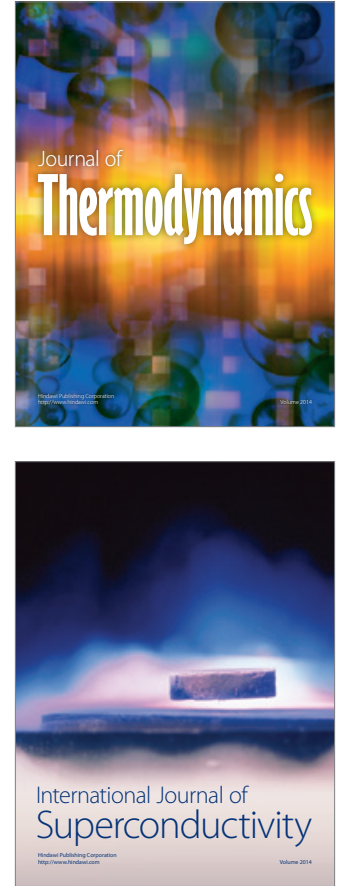
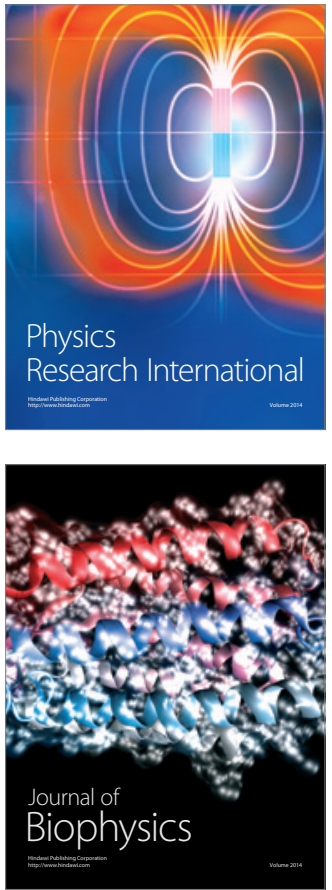
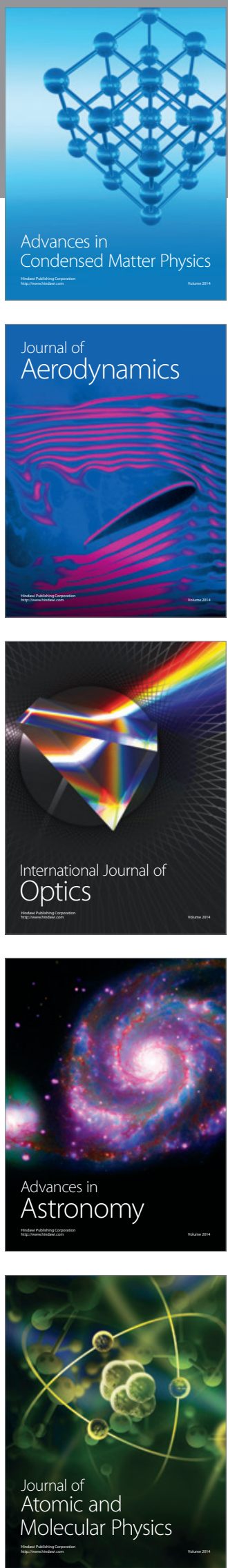